\title{
Interactive effects of aging and aerobic capacity on energy metabolism-related metabolites of serum, skeletal muscle, and white adipose tissue
}

\author{
Haihui Zhuang • Sira Karvinen (D) - Timo Törmäkangas • Xiaobo Zhang • \\ Xiaowei Ojanen • Vidya Velagapudi • Markku Alen • Steven L. Britton • \\ Lauren G. Koch • Heikki Kainulainen • Sulin Cheng • Petri Wiklund
}

Received: 21 July 2020 / Accepted: 17 May 2021 / Published online: 5 June 2021

(C) The Author(s) 2021

\begin{abstract}
Aerobic capacity is a strong predictor of longevity. With aging, aerobic capacity decreases concomitantly with changes in whole body metabolism leading to increased disease risk. To address the role of aerobic capacity, aging, and their interaction on metabolism, we utilized rat models selectively bred for low and high intrinsic aerobic capacity (LCRs/HCRs) and compared the metabolomics of serum, muscle, and white adipose tissue (WAT) at two time points: Young rats were
\end{abstract}

Haihui Zhuang, Sira Karvinen contributed equally (as first authors) and Heikki Kainulainen, Sulin Cheng and Petri Wiklund contributed equally (as senior authors).

H. Zhuang $\cdot$ X. Zhang $\cdot$ X. Ojanen $\cdot$ S. Cheng

School of Life Sciences and Biotechnology, Shanghai Jiao Tong University, Shanghai, China

H. Zhuang $\cdot$ X. Zhang $\cdot$ S. Cheng $\cdot$ P. Wiklund

Key Laboratory of Systems Biomedicine (Ministry of Education), and Exercise Translational Medicine Center, Shanghai Center for Systems Biomedicine, Shanghai Jiao Tong University, Shanghai, China

H. Zhuang · S. Karvinen $(\bowtie) \cdot$ T. Törmäkangas •

H. Kainulainen $\cdot$ S. Cheng

Faculty of Sport and Health Sciences, University of Jyväskylä, Jyväskylä, Finland

e-mail: sira.m.karvinen@jyu.fi

V. Velagapudi

Metabolomics Unit, Institute for Molecular Medicine Finland (FIMM), HiLIFE, University of Helsinki, Helsinki, Finland sacrificed at 9 months of age, and old rats were sacrificed at 21 months of age. Targeted and semiquantitative metabolomics analysis was performed on the ultra-pressure liquid chromatography tandem mass spectrometry (UPLC-MS) platform. The effects of aerobic capacity, aging, and their interaction were studied via regression analysis. Our results showed that high aerobic capacity is associated with an accumulation of isovalerylcarnitine in muscle and serum at rest, which is

M. Alen

Department of Medical Rehabilitation, Oulu University Hospital, Oulu, Finland

\section{S. L. Britton}

Department of Anesthesiology, University of Michigan, Ann Arbor, MI, USA

\section{S. L. Britton}

Molecular and Integrative Physiology, University of Michigan, Ann Arbor, MI, USA

L. G. Koch

Department of Physiology and Pharmacology, The University of Toledo College of Medicine and Life Sciences, Toledo, OH, USA

P. Wiklund Huawei Helsinki Research Center, Huawei Technologies Oy (Finland) Co. Ltd, Helsinki, Finland 
likely due to more efficient leucine catabolism in muscle. With aging, several amino acids were downregulated in muscle, indicating more efficient amino acid metabolism, whereas in WAT less efficient amino acid metabolism and decreased mitochondrial $\beta$-oxidation were observed. Our results further revealed that high aerobic capacity and aging interactively affect lipid metabolism in muscle and WAT, possibly combating unfavorable aging-related changes in whole body metabolism. Our results highlight the significant role of WAT metabolism for healthy aging.

Keywords Aerobic capacity · Aging · Metabolomics · Metabolites

\section{Introduction}

Epidemiological studies have indicated that low aerobic capacity, expressed as maximal oxygen uptake $\left(\mathrm{VO}_{2 \mathrm{max}}\right)$, is a major risk factor for cardio-metabolic diseases [1], whereas high aerobic capacity is associated with a more favorable cardio-metabolic health profile $[2,3]$, even in the presence of excess adiposity [4]. These observations are complemented by multiple randomized controlled trials, which have shown that exercise training significantly improves cardiorespiratory fitness and adiposity-related cardio-metabolic biomarkers [5]. Furthermore, aerobic capacity has been shown to be a strong independent predictor of cardiovascular and all-cause mortality in many population cohorts [6]. These studies suggest there is a fundamental connection between impaired aerobic metabolism, complex disease risk, and reduced longevity.

Epidemiological studies have also shown that aerobic capacity and physical activity level decrease with age [7, 8] concomitantly with quantitative and structural changes in body composition [9]. These changes are associated with alterations in whole body metabolism, insulin resistance, low-grade inflammation, and dyslipidemia, which contribute to the development of cardiometabolic disease with age $[10,11]$. However, it is not well understood to what extent the aging-related changes in metabolic profiles are attributable to either intrinsic aerobic capacity or decreased physical activity.

To enable the investigation of intrinsic aerobic endurance capacity on metabolic profile, Koch and Britton [12] developed heterogeneous rat lines by artificial selection for low and high inborn exercise capacity. This rat model of low capacity runners (LCRs) and high capacity runners (HCRs) prospectively tests the association between aerobic exercise capacity and survivability [13]. The phenotype of HCRs is coincident with a host of health benefits [14], including a $28-40 \%$ increased lifespan [15]. A recent study in HCRs and LCRs showed that an enhancement of aerobic capacity could mitigate some of the changes in the plasma metabolic profile, which were associated with aging [16]. Concomitantly with higher aerobic capacity, muscle mitochondrial function and oxidative energy metabolism are enhanced in HCRs compared with those in LCRs [17, 18]. In addition to skeletal muscle, white adipose tissue (WAT) shares an important role in the regulation of whole body metabolism [19] and WAT-to-muscle communication is critical in overall metabolic health [20].

Most previous studies have examined the effects of aerobic capacity on metabolism only in one tissue [21, 22] and using only young animals (3-4 months of age) [17]. Thus, possible interactive effects of aging and aerobic capacity on tissue metabolism are yet to be resolved. Here, we assessed metabolic profiles in serum, muscle, and WAT in both young ( 9 months of age) and old (21 months of age) HCR and LCR groups by using targeted and semi-quantitative metabolomics analysis which was performed on the triple quadrupole tandem mass spectrometry coupled to the ultra-pressure liquid chromatography (UPLC-MS) platform. We propose to define the most prominent differences for aerobic capacity in the metabolic profile of skeletal muscle [17, $18,23,24]$. Also, we expect that aerobic capacity and aging interactively affect several metabolites in muscle and WAT leading to changes in serum metabolite levels, as LCRs are known to have higher metabolic disease risk and shorter lifespan [13, 14].

\section{Materials and methods}

Animal model

The HCR/LCR rat model was derived from a genetically heterogeneous founder population (N:NIH stock) and bred with two-way artificial selection [12]. Animals were evaluated at 11 weeks of age for maximal running capacity at the University of Michigan (Ann Arbor, MI, USA) with a speed-ramped treadmill running test $\left(15^{\circ}\right.$ slope, initial velocity of $10 \mathrm{~m} / \mathrm{min}$, increased $1 \mathrm{~m} / \mathrm{min}$ every $2 \mathrm{~min}$ ). In this study, 34 female rats (16 HCRs and 
18 LCRs) from generations $23-27$ of selection were used. All rats were kept in an environmentally controlled facility with light/dark cycle of 12/12 $\mathrm{h}$ and had free access to food and tap water (R36, Labfor, Stockholm, Sweden).

\section{Testing procedure}

After arriving to Finland, rats were tested for maximal running capacity at the age of 9 months with the same speed-ramped running test as described previously [18]. Subsequently, both HCRs and LCRs were divided into weight and maximal running capacity-matched subgroups: HCR-Y or LCR-Y (young, $n=10$ in each group), and HCR-O (old, $n=6$ ) or LCR-O (old, $n=8$ ). After maximal aerobic capacity testing, HCR-Y and LCR-Y were weighed and sacrificed and hindlimb skeletal muscles, WAT, and blood samples were collected. HCR-O and LCR-O were continued to be housed individually in a standard cage until the age of 21 months. Maximal aerobic capacity was assessed again at the age of 21 months. After testing, animals were weighed and sacrificed and skeletal muscle, WAT, and serum samples were harvested.

Tissue collection

Soleus, extensor digitorum longus (EDL), plantaris, gastrocnemius, quadriceps femoris muscle, adipose tissue around the ovaries, visceral adipose tissue, and retroperitoneal adipose tissue were excised and weighed. Tissue samples were snap frozen in liquid nitrogen and then stored in $-80^{\circ} \mathrm{C}$ until analyses. Gastrocnemius, retroperitoneal adipose tissue, and serum were used for metabolomics analyses. Skeletal muscle mass was calculated as the sum weight of soleus, EDL, plantaris, gastrocnemius, and quadriceps femoris.

\section{Metabolomics analyses}

Targeted and semi-quantitative metabolomics analyses were performed on a Waters Xevo TQ-S triple quadrupole tandem mass spectrometer coupled to the ultrapressure liquid chromatography (UPLC-MS) platform using the previously published protocol in FIMM (Institute for Molecular Medicine Finland) [25]. Briefly, metabolites were extracted from $100 \mu \mathrm{L}$ serum, $20 \mathrm{mg}$ muscle, and $20 \mathrm{mg}$ WAT samples respectively using protein precipitation by adding acetonitrile $+1 \%$ formic acid. The collected extracts were dispensed in Ostro 96- well plates (Waters Corporation, Milford, USA) and filtered by applying a vacuum at a delta pressure of 300-400 mbar for $2.5 \mathrm{~min}$ on robot's vacuum station. Filtered sample extract $(5 \mu \mathrm{L})$ was injected in an Acquity UPLC system coupled to a Xevo TQ-S triple quadrupole mass spectrometer (Waters Corporation, Milford, MA, USA) which was operated in both positive and negative polarities with switching time of 20 ms. Multiple Reaction Monitoring (MRM) acquisition mode was selected for the quantification of metabolites. MassLynx 4.1 software was used for data acquisition, data handling, and instrument control. Data processing was done using TargetLynx 4.1 software. Eighty-nine metabolites in serum, 71 in muscle, and 71 in WAT were identified in whole samples.

\section{Statistical analysis}

The descriptive characteristics of rats were analyzed by using IBM SPSS Statistics 24.0 (SPSS, Chicago, USA). Data were checked for normality with the Shapiro-Wilk test. As most of the variables were not normally distributed, nonparametric tests were chosen for group comparisons, and statistical significance was set at $p<0.05$. The metabolomics analysis was same as in our previous study with serum and muscle samples [26].

All metabolomics data were transformed with a suitable transformation from the Box-Cox family and then scaled by dividing each variable with its standard deviation (unitvariance scaling, criteria for absolute value of the residuals for skew and kurtosis $<2$ ). We used partial-least-squares discriminant analysis (PLS-DA) for age and aerobic capacity group comparisons for assessing multivariate metabolite profiles. Permutation tests $(\mathrm{G}=200$ per model $)$ were used to validate the model and to avoid over-fitting.

We also performed comparative univariate analyses using linear regression models to assess (1) age (old vs. young) and aerobic capacity (high vs. low) group differences on metabolites, and (2) associations between metabolites and running speed while accounting also for the grouping structure (age and aerobic capacity). In the former case, we computed the $p$ value of the joint impact of both grouping factors (age and aerobic capacity) and their interaction. In the latter case, we used the $p$ value for the joint contribution of the metabolite-related predictors in the model (metabolite, metabolite $\times$ age, metabolite $\times$ aerobic capacity, metabolite $\times$ age $\times$ aerobic capacity). Model equations are shown in the Technical Supplement. In a PLS model, variable importance in 
projection (VIP) is used as an index of the estimated importance of a variable as a contributor to group separation in the model. Among metabolites, we selected metabolites based on VIP values $\geq 1$ and $p<0.05$ for the model combined impact of the factors in the univariate linear model.

For running speed-metabolite association, we considered as influential metabolites those with FDR-corrected $p$ value of the association with metabolite $<0.05$. Multivariate and univariate modeling were performed using a custom script in the $\mathrm{R}$ programming environment, version 4.0.2, utilizing packages psych (version 2.0.8) for assessment of normality of residuals and emmeans (version 1.5.1, https://CRAN.R-project.org/package=emmeans.) for computing estimated marginal coefficients and rolls (version 1.20.0) for PLS models [27].

Pathway analysis

MetaboAnalyst (v3.5) was used for pathway enrichment analysis to map significantly differential metabolites found in PLS-DA and regression models to their corresponding pathways $[28,29]$. This web-based tool relies on the knowledgebase of Kyoto Encyclopedia of Genes and Genomes (KEGG) metabolic pathway.

\section{Results}

Body mass, running capacity, and skeletal muscle mass/body mass ratio

HCRs had lower body mass and higher maximal running speed than LCRs both at 9 months $(p<0.001)$ and 21 months of age ( $p=0.002, p=0.006$, respectively) (Fig. 1a, c). Hindlimb skeletal muscle mass to body mass ratio was lower in both HCRs and LCRs when comparing 21month-old rats to 9-month-old (Fig. 1b). The maximal running speed was $25.5 \%$ lower in 21-month-old HCRs compared with 9-month-old HCRs $(p<0.001)$ whereas in LCRs, no significant difference in running speed with age was observed ( $p=0.360$; Fig. 1c).

Effects of intrinsic aerobic capacity and aging on metabolic profiles of serum, muscle, and white adipose tissue

We used PLS-DA to identify differences in the metabolic profiles between the rat lines (LCR vs. HCR) and age groups (young, Y vs. old, O). There were clear separations in serum $\left(Q^{2}=0.443, Q^{2}\right.$ intercept $=$ $-0.273)$, muscle $\left(Q^{2}=0.350, Q^{2}\right.$ intercept $\left.=-0.276\right)$, and WAT $\left(Q^{2}=0.325, Q^{2}\right.$ intercept $\left.=-0.405\right)$ metabolic profiles (Fig. 2; Table S1). However, a large difference between $R^{2}$ and $Q^{2}$ was observed in WAT tissue, suggesting a poor predictive performance of the model in WAT (Table S1). The corresponding associations between running speed and metabolites in groups of aerobic capacity and age are shown in Supplementary Table S4. When expressing the data via running speed, the metabolic profiles of serum and muscle are similar as with aerobic capacity, but the profile of WAT separated also at young age (Fig. S1).

Effects of intrinsic aerobic capacity and aging on single metabolites of serum, muscle, and white adipose tissue

Next, we compared the differences at the level of single metabolites between HCRs and LCRs with univariate analysis. The Venn diagrams of single metabolites changed by aerobic capacity, aging, and their interaction in serum, muscle, and WAT are shown in Figs. 3, 4, and 5. The corresponding univariate analyses with regression coefficients are shown in Supplementary Table S2 and design-controlled skew and kurtosis estimates for metabolites after most optimal transformation in Supplementary Table S5. Supplementary Table S3 shows the pathway analysis of the significantly changed metabolites.

Effect of intrinsic aerobic capacity on serum, muscle, and WAT metabolites

In serum, isovalerylcarnitine, inosine, and hexanoylcarnitine were upregulated with high aerobic capacity, whereas in muscle, only isovalerylcarnitine was upregulated (Figs 3 and 4; Table S2). In WAT, high aerobic capacity upregulated the level of dimethylglycine and downregulated the levels of betaine and octanoylcarnitine (Fig. 5; Table S2). The predominant pathways that were affected by aerobic capacity were nucleotide metabolism in serum and amino acid metabolism in WAT $(p<0.050$; Table S3).

Effect of aging on serum, muscle, and WAT metabolites

In serum, aging was associated with higher levels of several metabolites, having the most prominent effect 

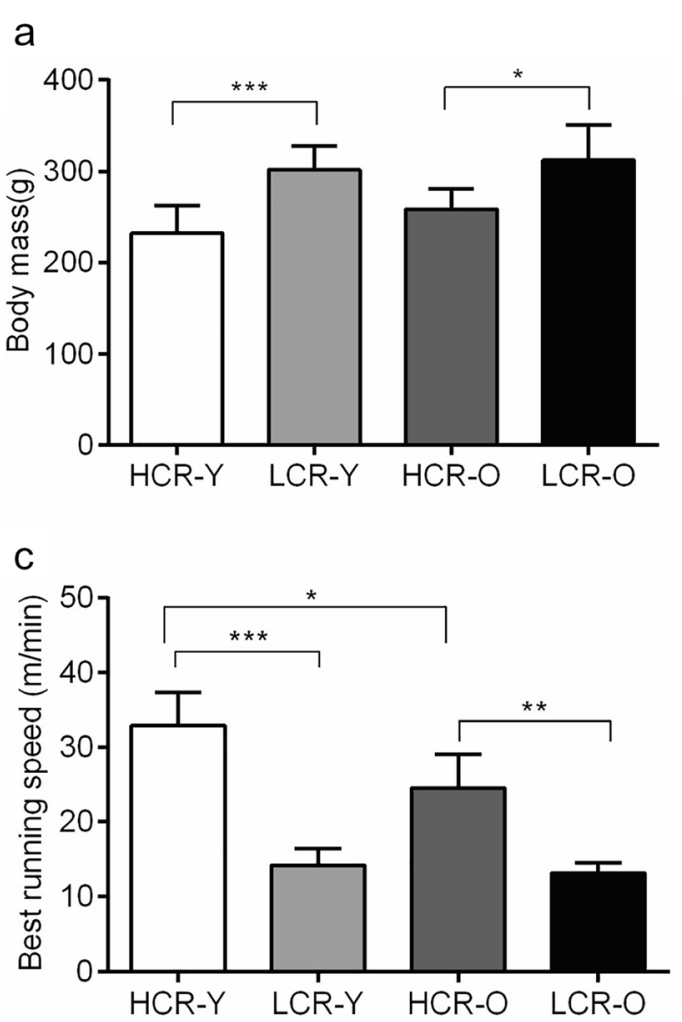

Fig. 1 The effects of aerobic capacity and aging on muscle mass to body mass ratio and maximal running speed in rats. Body mass (a), muscle mass to body mass ratio (b), and maximal running speed (c) in the studied rat groups. HCR-Y, high capacity runner,

on amino acid metabolites (Fig. 3; Table S2). The levels of isoleucine, proline, ornithine, and glutamine were downregulated, while alanine glycine citrulline, hydroxyproline, and aspartate were upregulated in serum (Fig. 3). In muscle, several amino acids were downregulated including branched-chain amino acids (BCAAs; valine, leucine, and isoleucine) (Fig. 4; Table S2). In WAT, alanine and leucine levels were upregulated and carnitine and several acylcarnitines (isobutyrylcarnitine, propionylcarnitine, and isovalerylcarnitine) were downregulated (Fig. 5; Table S2). The predominant pathways that were affected by aging were linked to amino acid metabolism in serum, muscle, and WAT $(p \leq 0.050$; Table S3).

\section{Effect of the interaction of aerobic capacity and aging on serum, muscle, and WAT metabolites}

We found that high aerobic capacity and aging interactively upregulated the levels of leucine, valine, arginine, isobutyrylcarnitine, and phosphoethanolamine, and b

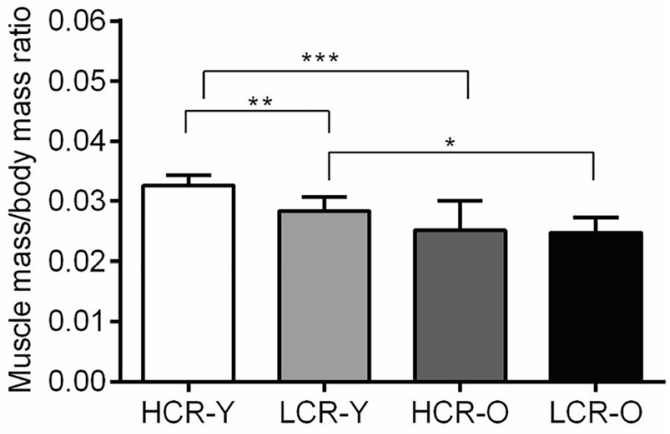

young; LCR-Y, low capacity runner, young; HCR-O, high capacity runner, old; LCR-O, low capacity runner, old. Data is presented as mean \pm SD. $* p<0.05, * * p<0.01$, and $* * * p<0.001$

downregulated succinate and uracil in serum (Fig. 3; Table S2). In muscle, acetylcarnitine and taurine were upregulated, whereas alanine, hydroxyproline, acetoacetic acid, succinate, ornithine, tryptophan, pantothenic acid, hippuric acid, folic acid, sucrose, and spermidine were downregulated (Fig. 4; Table S2). In WAT, aerobic capacity and aging interactively downregulated acetoacetic acid, citrulline, GABA, and AMP (Fig. 5; Table S2). The predominant pathways that were affected by the interaction of aerobic capacity and aging were linked to amino acid metabolism in serum and muscle and lipid metabolism in WAT $(p<0.050$; Table S3).

\section{Discussion}

The present study suggests that aerobic capacity and aging have different impacts on the metabolic profiles of serum, skeletal muscle, and white adipose tissue (WAT). Contrary to our hypothesis, only 


\section{Serum}
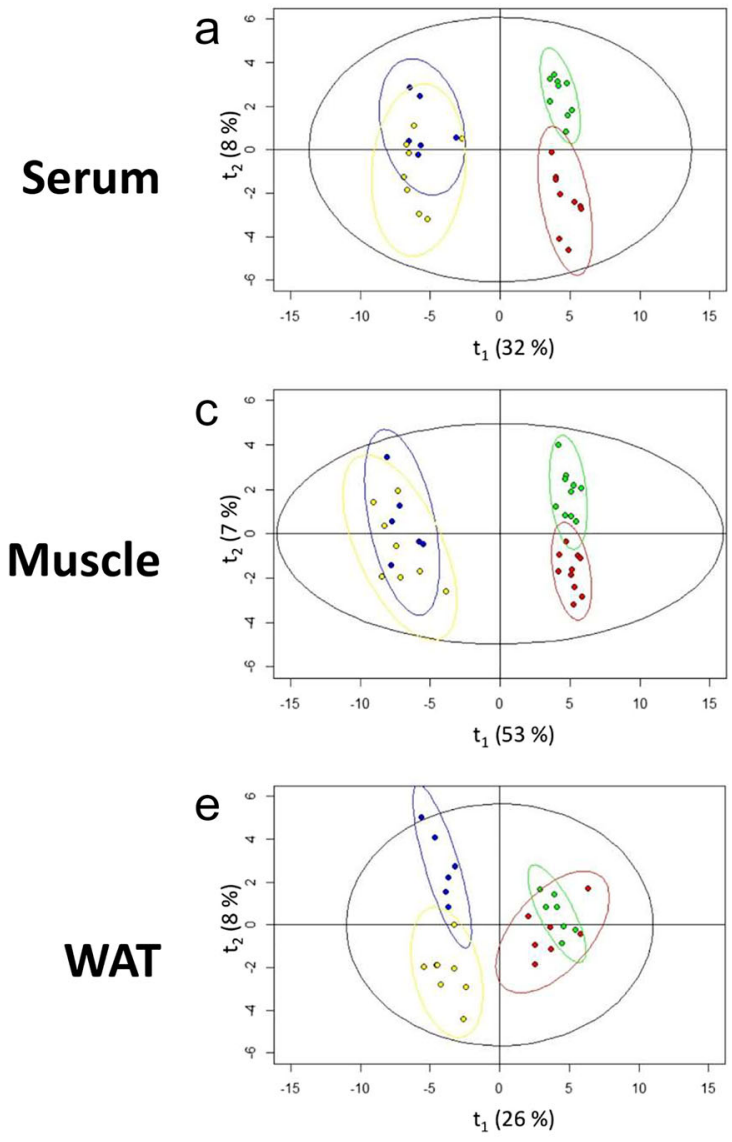

Fig. 2 Score plot of PLS-DA in serum (a), muscle (c), and WAT (e) with aerobic capacity and age and linear regression model with 200 times permutation test of the models for serum (b), muscle (d),

isovalerylcarnitine was upregulated by high aerobic capacity in muscle, whereas aging changed a large amount of metabolites predominantly linked to amino acid metabolism in serum, muscle, and WAT. Aerobic capacity and aging interactively affected several metabolites, the most predominant pathways being amino acid metabolism in serum and muscle and lipid metabolism in WAT.

Aerobic capacity and aging have distinct effects on metabolic profiles of muscle and WAT

We observed a clear separation of the metabolic profiles (PLS-DA) of serum and muscle in HCRs and LCRs at young age, whereas at old age, the metabolic profiles overlapped (HCR-Y vs. LCR-Y and HCR-O vs. LCR$\mathrm{O}$; Fig. 2). Interestingly, the opposite was observed for WAT, where the metabolic profiles overlapped at young

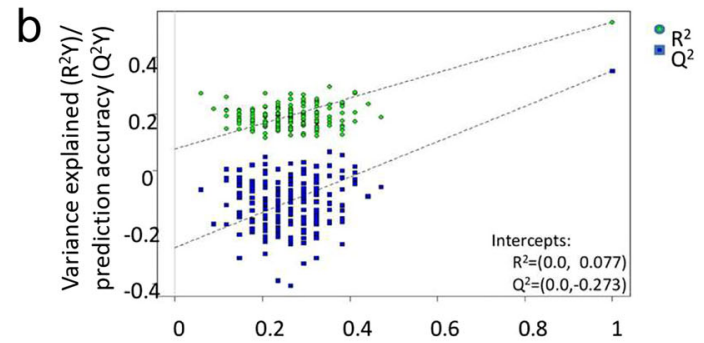

Observed-predicted metabolite correlation
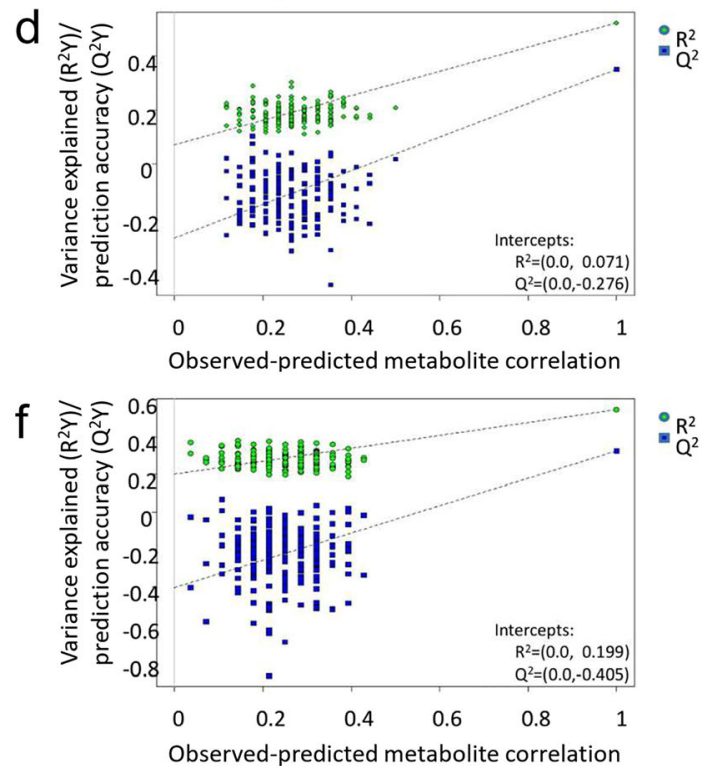

and WAT (f). LCR-O, low capacity runner, old; HCR-O, high capacity runner, old; LCR-Y, low capacity runner, young; HCR$\mathrm{Y}$, high capacity runner, young

age and showed a clear separation between HCRs and LCRs at old age (Fig. 2e). A previous study by Falegan et al. examined the metabolic profile of plasma of young (13 months) and old (26 months) male HCR and LCR rats and observed a strong profile separation in old and LCRs, whereas young and HCRs were found less predictive [16]. Hence, they concluded that in plasma, metabolomics analysis better predicts age rather than aerobic capacity. Yet our results from serum suggest that at old age, the metabolic profiles of HCRs and LCRs are more similar. The reason for the divergence in observations may be due to differences in sex, age, and generation (Falegan et al. used rats from generations 17 and 19 of selection), fasting duration, and euthanasia method. Different metabolomics platforms may also account for the discrepant results as Falegan et al. used NMR proton spectrometry, while in the present study 


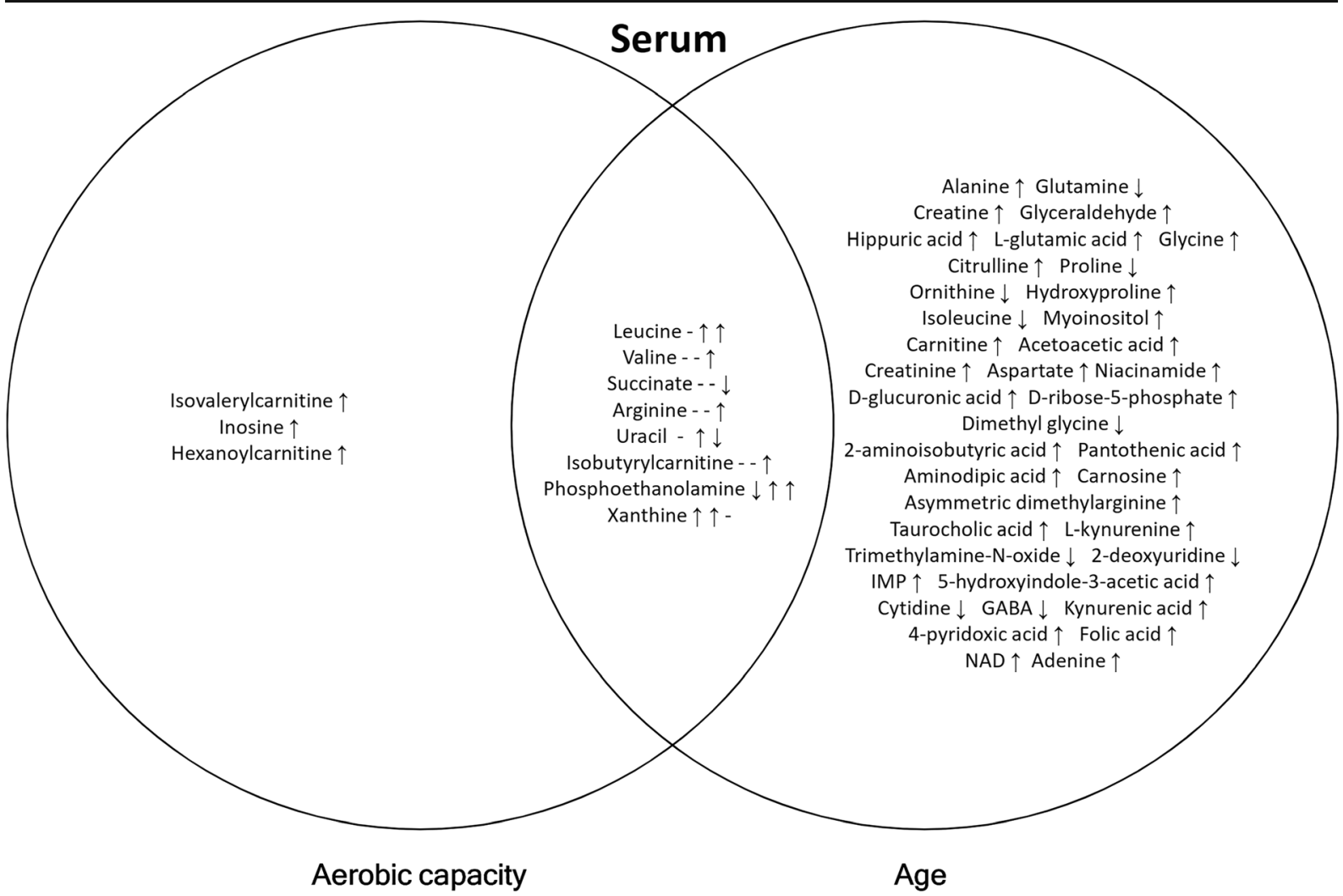

Fig. 3 Venn diagram of differentially expressed metabolites with aerobic capacity, age, and their interaction in serum. Arrows represent the direction of regression coefficient; that in the

we used UPLC-MS in targeted metabolite profiling. Nevertheless, our results highlight the role of WAT metabolism in healthy aging.

We further observed that the metabolic profiles of serum and muscle were similar irrespective of whether the data was expressed using the rat lines and age groups (Fig. 2a, c) or via running speed (Fig. S1a, c). Our result suggests that the separation of the rat lines through maximal running capacity has driven the changes in the metabolism of these rats, as high running speed is the practical manifestation of the genetic breeding of HCR rats and LCR rats. Interestingly, unlike in the line vs. age comparison, the metabolic profiles of young rats appeared differentially expressed when shown via running speed (Fig. S1e). This observation may be due to a large difference in the running speed between the rat lines at young age (HCR-Y vs. LCR-Y), but that is less prominent at old age (Fig. 1c). These observations suggest that aerobic capacity enhances the metabolism of adipose tissue and supports earlier studies which have shown that exercise training enhances white adipose overlapping region (aerobic capacity * age) may originate from aerobic capacity (1st arrow) and age (2nd arrow) or their interaction (3rd arrow)

tissue metabolism in HCR and LCR rats [30], and that adipose tissue may mediate some of the health benefits of aerobic exercise training [31, 32].

High aerobic capacity is associated with efficient leucine catabolism in muscle and altered amino acid and fatty acid metabolism in WAT

Skeletal muscle is an important tissue contributing both to aerobic capacity and whole body metabolism [33]. Hence, it was unexpected that high aerobic capacity was associated only with upregulation of isovalerylcarnitine in muscle (Fig. 3). Isovalerylcarnitine is produced during leucine catabolism and accumulation of isovalerylcarnitine in serum might indicate a defect in leucine catabolism $[34,35]$. In humans, increased concentrations of isovalerylcarnitine and hexanoylcarnitine have been previously reported in type 2 diabetes [36-38] as well as in a model of type 2 diabetesZucker diabetic fatty rats [39]. However, efficient leucine catabolism may also lead to accumulation of 


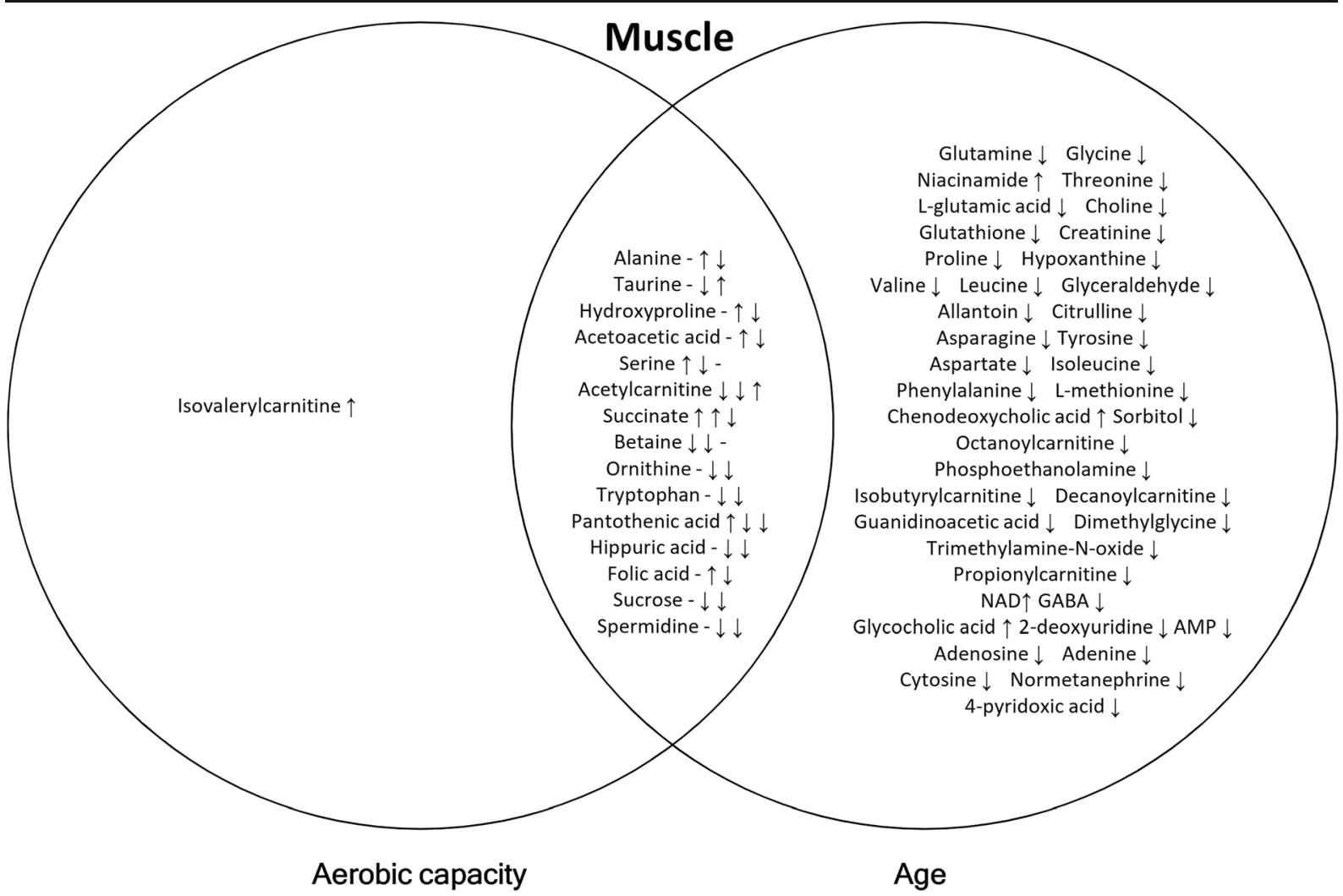

Fig. 4 Venn diagram of differentially expressed metabolites with aerobic capacity, age, and their interaction in muscle. Arrows represent the direction of regression coefficient; that in the

isovalerylcarnitine when energy demand decreases. HCRs may catabolize leucine more actively during movement to supply acetyl-coenzyme A to tricarboxylic acid cycle, whereas at rest the produced isovalerylcoenzyme A is not needed, and is further metabolized to isovalerylcarnitine. Supporting this hypothesis, our previous studies have shown increased expression of the enzymes of BCAA catabolism in skeletal muscle of HCRs [24] and physically active humans [40]. Also, a previous study by Overmyer et al. showed that young (3-4.5 months of age) HCRs oxidize BCAAs (leucine, isoleucine, and valine) more efficiently compared to LCRs of the same age [17]. Furthermore, they found that muscle BCAAs were lower in HCRs than in LCRs after $10 \mathrm{~min}$ of speed-ramped treadmill running. These changes were paralleled by a fall in plasma BCAAs in HCRs, indicating increased utilization of BCAAs during exercise. According to our results, we speculate that during resting at fasted state, HCRs may accumulate isovalerylcarnitine into muscle and serum due to more efficient leucine catabolism in muscle. overlapping region (aerobic capacity * age) may originate from aerobic capacity (1st arrow) and age (2nd arrow) or their interaction (3rd arrow) or both

Comparedwith other metabolically active tissues, the oxidative capacity of WAT is relatively low. For instance, $\mathrm{O}_{2}$ consumption per kg wet weight in WAT is about onetenth of that of the resting skeletal muscle [41]. In WAT tissue, high aerobic capacity upregulated dimethylglycine and downregulated betaine (trimethylglycine) (Fig. 5) suggesting increased methylation of homocysteine to methionine, which has various functions in metabolism [42]. However, the possible role of betaine in HCR WAT remains to be shown. Octanoylcarnitine was also downregulated (Fig. 5) suggesting more efficient fatty acid oxidation with higher aerobic capacity in WAT. We observed a difference only in three metabolites, indicating relatively small differences between HCR and LCR rats in WAT metabolism.

Aging was associated with the most prominent changes in serum, muscle, and WAT metabolites

In our study setup, aging was associated with the most prominent changes in serum, muscle, and WAT (Figs. 3, 4, 


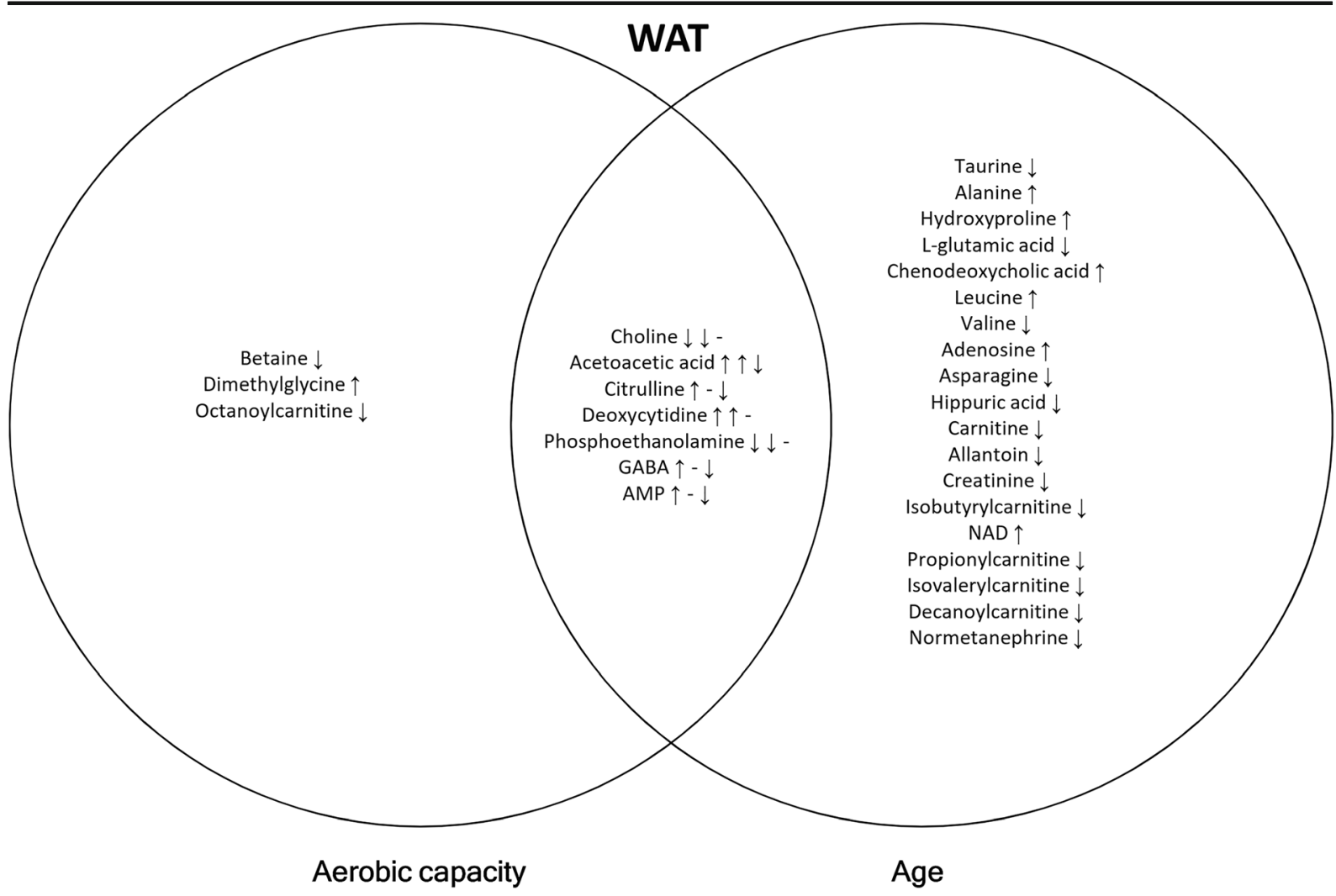

Fig. 5 Venn diagram of differentially expressed metabolites with aerobic capacity, age, and their interaction in WAT. Arrows represent the direction of regression coefficient; that in the overlapping region (aerobic capacity * age) may originate from aerobic capacity (1st arrow) and age (2nd arrow) or their interaction (3rd arrow) and 5) supporting previous findings that highlighted the importance of age when investigating metabolic changes in aerobic capacity [16]. In serum, aging was associated with higher level of several metabolites, having the most noticeable effect on amino acid metabolites (Fig. 3; Table S3). The levels of isoleucine, proline, ornithine, and glutamine were downregulated, while citrulline, alanine, glycine, hydroxyproline, and aspartate were upregulated in serum (Fig. 3). With aging, the serum concentrations of amino acids typically decrease. This is mainly related to the reduction of dietary protein intake and alterations in gluconeogenesis and the function of urea cycle $[43,44]$. Upregulated citrulline and alanine levels in serum may indicate less efficient urea cycle.

In muscle, the levels of several amino acids were downregulated, including BCAAs, indicating more efficient amino acid metabolism with aging. In human, basal amino acid metabolism may be unaffected by age, yet old animals and humans appear to have a decreased ability to respond to anabolic stimuli $[45,46]$. Our results suggest that certain metabolic pathways activate with aging, possibly combating the unfavorable changes in skeletal muscle metabolism and loss of muscle mass that occurs with aging [47].

In contrast, in WAT, alanine and leucine levels were upregulated indicating less efficient amino acid metabolism with aging (Fig. 5). This observation is in agreement with previous studies suggesting that adipose tissue plays a central role in the development of insulin resistance, metabolic dysfunction, inflammation, and impaired regenerative capacity during aging [48-50]. In addition, WAT carnitine and several acylcarnitines (isobutyrylcarnitine, propionylcarnitine, and isovalerylcarnitine) were downregulated with aging. Acylcarnitines transport fatty acids into mitochondria and are essential for $\beta$-oxidation and, hence, energy metabolism [51]. Downregulation of carnitine and acylcarnitines in WAT suggests a decreased mitochondrial $\beta$-oxidation, which may contribute to aging-related fat accumulation. 
Interaction of aerobic capacity and aging alters lipid metabolism in muscle and WAT

Previous human studies propose that high aerobic capacity reduces symptoms of age-related disorders, including obesity, diabetes, inflammation, and cardiovascular diseases $[52,53]$, suggesting that the interaction of aerobic capacity and aging has a combined effect on metabolism. In the present study, high aerobic capacity and aging interactively upregulated the levels of leucine and valine in serum (Fig. 3; Table S3), indicating less efficient BCAA utilization from serum at rest with high aerobic capacity and aging. It should be noted that during aging, aerobic capacity of HCR rats declines more than that of LCRs (Fig. 1), which may at least partially explain this interaction. In turn, in muscle, several amino acids were downregulated (alanine, hydroxyproline, ornithine, and tryptophan) (Fig. 4; Table S3), suggesting an alteration in amino acid metabolism with higher aerobic capacity and aging. Also, acetoacetic acid, succinate, and pantothenic acid linked to lipid metabolism - more precisely to acetyl-CoA formation from liver-originating acetoacetate - were downregulated in muscle, indicating that interaction of aerobic capacity and aging reduces mitochondrial energy metabolism in this tissue. Although the role of ketone bodies in skeletal muscle metabolism is limited, it has been found both in animal and human studies that ketogenic diet increases mitochondrial energy metabolism, especially fat oxidation [54, 55]. In WAT, aerobic capacity and aging interactively downregulated acetoacetic acid, citrulline, GABA, and AMP, further referring to altered lipid metabolism (Fig. 5; Table S3). Our results suggest that high aerobic capacity and advanced age interactively affect lipid metabolism in muscle and WAT, possibly combating aging-related unfavorable changes in the whole body metabolism as well as fat accumulation.

\section{Conclusion}

Our results suggest that high aerobic capacity is associated with an accumulation of isovalerylcarnitine into muscle and serum at rest, which is likely due to more efficient leucine catabolism in muscle. Aerobic capacity also altered amino acid and fatty acid metabolism in WAT, although significant difference was observed only in three metabolites. In our study setup, aging was associated with the most prominent changes in metabolites. In muscle, several amino acids were downregulated, suggesting more efficient amino acid metabolism with aging. In contrast, the results from WAT indicated less efficient amino acid metabolism and decreased mitochondrial $\beta$-oxidation with aging. Our results further revealed that high aerobic capacity and age interact affecting lipid metabolism in muscle and WAT, possibly combating aging-related unfavorable changes in whole body metabolism as well as fat accumulation. Our results highlight the role of WAT metabolism in healthy aging. Future studies are needed to determine whether improved aerobic capacity by training could restore the observed aging-related metabolic changes.

Supplementary Information The online version contains supplementary material available at https://doi.org/10.1007/s11357021-00387-1.

Code availability Not applicable.

Author contribution Haihui Zhuang performed the original statistical analysis and drafted the first version of the manuscript. Sira Karvinen performed the animal experiment and sample harvest and drafted the revised version of the manuscript. Xiaobo Zhang, Xiaowei Ojanen, and Markku Alen edited the first version of the manuscript. Vidya Velagapudi performed the metabolomics analysis. Timo Törmäkangas designed and ran the statistical analysis of the revised version of the manuscript and participated in drafting the revised version of the manuscript. Steven L. Britton and Lauren G. Koch developed and provided the animal models for the study. Heikki Kainulainen, Sulin Cheng, and Petri Wiklund designed the study setup and supervised all steps of the process. All authors read and approved the final version of the manuscript.

Funding Open access funding provided by University of Jyväskylä (JYU). This study was funded by the Academy of Finland (grant no. 298875 to HK), Biocenter Finland, and HILIFE (VV). Finnish Government Scholarship Pool - academic year 2019/2020 (ZH), EVO research grants (years 2014/2015) from Oulu University Hospital (MA), Shanghai Jiao Tong University 111 Project (B17029, SC), Shanghai Jiao Tong University Zhiyuan Foundation (grant CP2014013, SC), and China Postdoc Scholarship Council (201806230001, XO). The LCR and HCR rat models are funded by the Office of Infrastructure Programs grant P40ODO21331 (to L.G.K and S.L.B) from the National Institutes of Health. These rat models for low and high intrinsic exercise capacity are maintained as an international resource with support from the Department of Physiology \& Pharmacology, The University of Toledo College of Medicine, Toledo, OH. Contact L.G.K Lauren.Koch2@UToledo.edu or S.L.B brittons@umich.edu for information on the rat models.

Data Availability The data set is now deposited to MetaboLights https://www.ebi.ac.uk/metabolights/MTBLS2234 /descriptors.Supplementary Information The online version contains supplementary material available at https://doi. org/10.1007/s11357-021-00387-1. 


\section{Declarations}

Ethics approval This study was approved by the National Animal Experiment Board, Finland (permit number ESAVI-201007989/Ym-23).

\section{Consent to participate Not applicable.}

Consent for publication All authors agree on consent of publication.

Conflict of interest The authors declare no competing interests.

Open Access This article is licensed under a Creative Commons Attribution 4.0 International License, which permits use, sharing, adaptation, distribution and reproduction in any medium or format, as long as you give appropriate credit to the original author(s) and the source, provide a link to the Creative Commons licence, and indicate if changes were made. The images or other third party material in this article are included in the article's Creative Commons licence, unless indicated otherwise in a credit line to the material. If material is not included in the article's Creative Commons licence and your intended use is not permitted by statutory regulation or exceeds the permitted use, you will need to obtain permission directly from the copyright holder. To view a copy of this licence, visit http://creativecommons.org/licenses/by/4.0/.

\section{References}

1. Ross R, Blair SN, Arena R, Church TS, Despres JP, Franklin $\mathrm{BA}$, et al. Importance of assessing cardiorespiratory fitness in clinical practice: a case for fitness as a clinical vital sign: a scientific statement from the American Heart Association. Circulation. 2016;134(24):e653-99.

2. Lee DC, Sui X, Church TS, Lavie CJ, Jackson AS, Blair SN. Changes in fitness and fatness on the development of cardiovascular disease risk factors hypertension, metabolic syndrome, and hypercholesterolemia. J Am Coll Cardiol. 2012;59(7):665-72.

3. Fogelholm M. Physical activity, fitness and fatness: relations to mortality, morbidity and disease risk factors. A systematic review. Obes Rev. 2010;11(3):202-21.

4. Barry VW, Baruth M, Beets MW, Durstine JL, Liu J, Blair SN. Fitness vs. fatness on all-cause mortality: a meta-analysis. Prog Cardiovasc Dis. 2014;56(4):382-90.

5. Lin X, Zhang X, Guo J, Roberts CK, McKenzie S, Wu WC, et al. Effects of exercise training on cardiorespiratory fitness and biomarkers of cardiometabolic health: a systematic review and meta-analysis of randomized controlled trials. J Am Heart Assoc. 2015;4(7). https://doi.org/10.1161 /JAHA.115.002014.

6. Harber MP, Kaminsky LA, Arena R, Blair SN, Franklin BA, Myers $\mathrm{J}$, et al. Impact of cardiorespiratory fitness on allcause and disease-specific mortality: advances since 2009 . Prog Cardiovasc Dis. 2017;60(1):11-20.
7. Bouchard C, Blair SN, Katzmarzyk PT. Less sitting, more physical activity, or higher fitness? Mayo Clin Proc. 2015 Nov;90(11):1533-40.

8. Coffman KE, Carlson AR, Miller AD, Johnson BD, Taylor BJ. The effect of aging and cardiorespiratory fitness on the lung diffusing capacity response to exercise in healthy humans. J Appl Physiol (1985). 2017;122(6):1425-34.

9. Beaufrere B, Morio B. Fat and protein redistribution with aging: metabolic considerations. Eur J Clin Nutr. 2000;54(Suppl 3):S48-53.

10. Floegel A, Wientzek A, Bachlechner U, Jacobs S, Drogan D, Prehn C, et al. Linking diet, physical activity, cardiorespiratory fitness and obesity to serum metabolite networks: findings from a population-based study. Int J Obes. 2014;38(11): 1388-96.

11. Bouchard DR, McGuire KA, Davidson L, Ross R. Cardiorespiratory fitness, obesity, and functional limitation in older adults. J Aging Phys Act. 2011;19(4):336-46.

12. Koch LG, Britton SL. Artificial selection for intrinsic aerobic endurance running capacity in rats. Physiol Genomics. 2001;5(1):45-52.

13. Koch LG, Britton SL, Wisloff U. A rat model system to study complex disease risks, fitness, aging, and longevity. Trends Cardiovasc Med. 2012;22(2):29-34.

14. Wisloff U, Najjar SM, Ellingsen O, Haram PM, Swoap S, Al-Share Q, et al. Cardiovascular risk factors emerge after artificial selection for low aerobic capacity. Science. 2005;307(5708):418-20.

15. Koch LG, Kemi OJ, Qi N, Leng SX, Bijma P, Gilligan LJ, et al. Intrinsic aerobic capacity sets a divide for aging and longevity. Circ Res. 2011;109(10):1162-72.

16. Falegan OS, Vogel HJ, Hittel DS, Koch LG, Britton SL, Hepple RT, et al. High aerobic capacity mitigates changes in the plasma metabolomic profile associated with aging. J Proteome Res. 2017;16(2):798-805.

17. Overmyer KA, Evans CR, Qi NR, Minogue CE, Carson JJ, Chermside-Scabbo CJ, et al. Maximal oxidative capacity during exercise is associated with skeletal muscle fuel selection and dynamic changes in mitochondrial protein acetylation. Cell Metab. 2015;21(3):468-78.

18. Karvinen SM, Silvennoinen M, Ma H, Tormakangas T, Rantalainen T, Rinnankoski-Tuikka R, et al. Voluntary running aids to maintain high body temperature in rats bred for high aerobic capacity. Front Physiol. 2016;7:311.

19. Heinonen S, Jokinen R, Rissanen A, Pietilainen KH. White adipose tissue mitochondrial metabolism in health and in obesity. Obes Rev. 2020;21(2):e12958.

20. Stanford KI, Goodyear LJ. Muscle-adipose tissue cross talk. Cold Spring Harb Perspect Med. 2018;8(8). https://doi. org/10.1101/cshperspect.a029801.

21. Wone B, Donovan ER, Hayes JP. Metabolomics of aerobic metabolism in mice selected for increased maximal metabolic rate. Comp Biochem Physiol D Genomics Proteomics. 2011;6(4):399-405.

22. Bowden-Davies K, Connolly J, Burghardt P, Koch LG, Britton SL, Burniston JG. Label-free profiling of white adipose tissue of rats exhibiting high or low levels of intrinsic exercise capacity. Proteomics. 2015;15(13):2342-9.

23. Karvinen S, Silvennoinen M, Vainio P, Sistonen L, Koch LG, Britton SL, et al. Effects of intrinsic aerobic capacity, 
aging and voluntary running on skeletal muscle sirtuins and heat shock proteins. Exp Gerontol. 2016;79:46-54.

24. Kivela R, Silvennoinen M, Lehti M, Rinnankoski-Tuikka R, Purhonen T, Ketola T, et al. Gene expression centroids that link with low intrinsic aerobic exercise capacity and complex disease risk. FASEB J. 2010;24(11):4565-74.

25. Nandania J, Peddinti G, Pessia A, Kokkonen M, Velagapudi V. Validation and automation of a high-throughput multitargeted method for semiquantification of endogenous metabolites from different biological matrices using tandem mass spectrometry. Metabolites. 2018;8(3). https://doi. org/10.3390/metabo8030044

26. Lautaoja JH, Lalowski M, Nissinen TA, Hentila J, Shi Y, Ritvos O, et al. Muscle and serum metabolomes are dysregulated in colon-26 tumor-bearing mice despite amelioration of cachexia with activin receptor type $2 \mathrm{~B}$ ligand blockade. Am J Physiol Endocrinol Metab. 2019;316(5):E852-65.

27. Thevenot EA, Roux A, Xu Y, Ezan E, Junot C. Analysis of the human adult urinary metabolome variations with age, body mass index, and gender by implementing a comprehensive workflow for univariate and OPLS statistical analyses. J Proteome Res. 2015;14(8):3322-35.

28. Xia J, Sinelnikov IV, Han B, Wishart DS. MetaboAnalyst 3.0-making metabolomics more meaningful. Nucleic Acids Res. 2015;43(W1):W251-7.

29. Xia J, Wishart DS. Using MetaboAnalyst 3.0 for comprehensive metabolomics data analysis. Curr Protoc Bioinformatics. 2016;55:14.10.1,14.10.91.

30. Stephenson EJ, Lessard SJ, Rivas DA, Watt MJ. Yaspelkis BB,3rd, Koch LG, et al. Exercise training enhances white adipose tissue metabolism in rats selectively bred for low- or high-endurance running capacity. Am J Physiol Endocrinol Metab. 2013;305(3):E429-38.

31. Stanford KI, Middelbeek RJ, Townsend KL, Lee MY, Takahashi H, So K, et al. A novel role for subcutaneous adipose tissue in exercise-induced improvements in glucose homeostasis. Diabetes. 2015;64(6):2002-14.

32. Vidal P, Stanford KI. Exercise-induced adaptations to adipose tissue thermogenesis. Front Endocrinol (Lausanne). 2020;11:270.

33. Argiles JM, Campos N, Lopez-Pedrosa JM, Rueda R, Rodriguez-Manas L. Skeletal muscle regulates metabolism via interorgan crosstalk: roles in health and disease. J Am Med Dir Assoc. 2016;17(9):789-96.

34. Adeva-Andany MM, Lopez-Maside L, Donapetry-Garcia C, Fernandez-Fernandez C, Sixto-Leal C. Enzymes involved in branched-chain amino acid metabolism in humans. Amino Acids. 2017;49(6):1005-28.

35. Koves TR, Ussher JR, Noland RC, Slentz D, Mosedale M, Ilkayeva $\mathrm{O}$, et al. Mitochondrial overload and incomplete fatty acid oxidation contribute to skeletal muscle insulin resistance. Cell Metab. 2008;7(1):45-56.

36. Mai M, Tonjes A, Kovacs P, Stumvoll M, Fiedler GM, Leichtle AB. Serum levels of acylcarnitines are altered in prediabetic conditions. PLoS One. 2013;8(12):e82459.

37. Adams SH, Hoppel CL, Lok KH, Zhao L, Wong SW, Minkler PE, et al. Plasma acylcarnitine profiles suggest incomplete long-chain fatty acid beta-oxidation and altered tricarboxylic acid cycle activity in type 2 diabetic AfricanAmerican women. J Nutr. 2009;139(6):1073-81.

38. Mihalik SJ, Goodpaster BH, Kelley DE, Chace DH, Vockley J, Toledo FG, et al. Increased levels of plasma acylcarnitines in obesity and type 2 diabetes and identification of a marker of glucolipotoxicity. Obesity (Silver Spring). 2010;18(9):1695-700.

39. She P, Olson KC, Kadota Y, Inukai A, Shimomura Y, Hoppel CL, et al. Leucine and protein metabolism in obese Zucker rats. PLoS One. 2013;8(3):e59443.

40. Leskinen T, Rinnankoski-Tuikka R, Rintala M, SeppanenLaakso T, Pollanen E, Alen M, et al. Differences in muscle and adipose tissue gene expression and cardio-metabolic risk factors in the members of physical activity discordant twin pairs. PLoS One. 2010;5(9). https://doi.org/10.1371/journal.pone.0012609.

41. Frayn KN, Humphreys SM, Coppack SW. Fuel selection in white adipose tissue. Proc Nutr Soc. 1995 Mar;54(1):177-89.

42. Elango R. Methionine nutrition and metabolism: insights from animal studies to inform human nutrition. J Nutr. 2020;150(Supplement_1):2518S-23S.

43. Kouchiwa T, Wada K, Uchiyama M, Kasezawa N, Niisato $\mathrm{M}$, Murakami H, et al. Age-related changes in serum amino acids concentrations in healthy individuals. Clin Chem Lab Med. 2012;50(5):861-70.

44. Le Couteur DG, Ribeiro R, Senior A, Hsu B, Hirani V, Blyth FM, et al. Branched chain amino acids, cardiometabolic risk factors and outcomes in older men: the Concord Health and Ageing in Men Project. J Gerontol A Biol Sci Med Sci. 2020;75(10):1805-10.

45. Mosoni L, Valluy MC, Serrurier B, Prugnaud J, Obled C, Guezennec CY, et al. Altered response of protein synthesis to nutritional state and endurance training in old rats. Am J Phys. 1995;268(2 Pt 1):E328-35.

46. Timmerman KL, Volpi E. Amino acid metabolism and regulatory effects in aging. Curr Opin Clin Nutr Metab Care. 2008;11(1):45-9.

47. Koopman R, van Loon LJ. Aging, exercise, and muscle protein metabolism. J Appl Physiol (1985). 2009;106(6): 2040-8.

48. Tchkonia T, Morbeck DE, Von Zglinicki T, Van Deursen J, Lustgarten J, Scrable H, et al. Fat tissue, aging, and cellular senescence. Aging Cell. 2010;9(5):667-84.

49. Palmer AK, Kirkland JL. Aging and adipose tissue: potential interventions for diabetes and regenerative medicine. Exp Gerontol. 2016;86:97-105.

50. De Carvalho FG, Justice JN, Freitas EC, Kershaw EE, Sparks LM. Adipose tissue quality in aging: how structural and functional aspects of adipose tissue impact skeletal muscle quality. Nutrients. 2019;11(11). https://doi. org/10.3390/nu11112553.

51. Bremer J. Carnitine-metabolism and functions. Physiol Rev. 1983;63(4):1420-80.

52. Kokkinos P, Doumas M, Myers J, Faselis C, Manolis A, Pittaras A, et al. A graded association of exercise capacity and all-cause mortality in males with high-normal blood pressure. Blood Press. 2009;18(5):261-7. 
53. Thompson PD, Buchner D, Pina IL, Balady GJ, Williams MA, Marcus BH, et al. Exercise and physical activity in the prevention and treatment of atherosclerotic cardiovascular disease: a statement from the Council on Clinical Cardiology (Subcommittee on Exercise, Rehabilitation, and Prevention) and the Council on Nutrition, Physical Activity, and Metabolism (Subcommittee on Physical Activity). Circulation. 2003;107(24):3109-16.

54. Miller VJ, Villamena FA, Volek JS. Nutritional ketosis and mitohormesis: potential implications for mitochondrial function and human health. J Nutr Metab. 2018;2018:5157645.
55. Miller VJ, LaFountain RA, Barnhart E, Sapper TS, Short J, Arnold WD, et al. A ketogenic diet combined with exercise alters mitochondrial function in human skeletal muscle while improving metabolic health. Am J Physiol Endocrinol Metab. 2020;319(6):E995-E1007.

Publisher's note Springer Nature remains neutral with regard to jurisdictional claims in published maps and institutional affiliations. 